\title{
Model Error Concepts in Identification for Control
}

\author{
Wolfgang Reinelt*, Andrea Garulli**, Lennart Ljung*, Julio H. Braslavsky*** and Antonio \\ Vicino** \\ *Department of Electrical Engineering \\ Linköping University, 58183 Linköping, Sweden \\ WWW: http://www.control.isy.liu.se/ \\ Email: \{wolle, ljung\}@isy.liu.se \\ **Dipartimento di Ingegneria dell'Informazione \\ Universitá di Siena, 53100 Siena,Italy \\ WWW: http://www-dii.ing. unisi.it/ control/ \\ Email: $\{$ garulli, vicino\}@ing.unisi.it \\ *** Depto de Ciencia y Tecnologia \\ Universidad Nacional de Quilmes, 1876 Bernal, Argentina \\ WWW: http://www.unq.edu.ar/ \\ Email: jbrasla@unq.edu.ar
}

August 1999

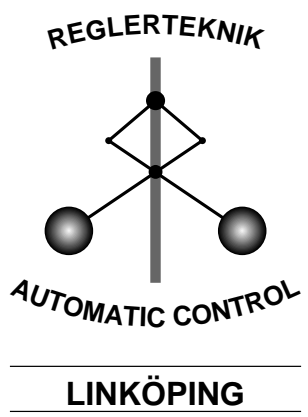

Report no.: LiTH-ISY-R-2188

Submitted to the 38th IEEE Conference on Decision and Control, Phoenix, AZ, USA (December 4-7, 1999). Invited Session on Model Set Theory for Robust Identification and Control, organized by Mario Milanese and Hidenori Kimura.

Technical reports from the Automatic Control group in Linköping are available by anonymous ftp at the address ftp.control.isy.liu.se. This report is contained in the portable document format file 2188. pdf. 


\title{
Model Error Concepts in Identification for Control*
}

\author{
Wolfgang Reinelt*, Andrea Garulli*, Lennart Ljung*, \\ Julio H. Braslavsky*** and Antonio Vicino** \\ *Dept of Electrical Engineering, University of Linköping \\ S-581 83 Linköping, Sweden \\ E-mail: \{wolle, ljung\}@isy.liu.se \\ **Dipartimento di Ingegneria dell'Informazione, Universitá di Siena, \\ I-53100 Siena, Italy \\ E-mail: \{garulli,vicino\}@ing.unisi.it \\ *** Depto de Ciencia y Tecnologia, Universidad Nacional de Quilmes, \\ 1876 Bernal, Argentina \\ E-mail: jbrasla@unq.edu.ar
}

\begin{abstract}
We examine some recent methods for System Identification, that also deliver non-parametric error bounds, suited for a robust controller design. In particular, we look at Stochastic Embedding, Set Membership Identification and Model Error Modelling. We briefly review the main ideas together with existing computational solutions and present a comparative example.
\end{abstract}

Keywords: Identification for Robust Control, Stochastic Embedding, Set Membership Identification, Model Error Modelling.

\section{Introduction and Motivation}

Identification for control is an area which has received a renewed interest since the beginning of the 1990s (see the special issues [7, 10]) and still attracts a growing number of researchers (see [5] for a recent collection of papers and references). A major motivation of this research is to achieve robust stabilization of the real plant. Thus it is customary to identify not only a nominal model, but also an uncertainty set, i.e. a set of models to be considered in the control design process. For example, if non-parametric design methods are adopted, it is natural to look for uncertainty regions in the Bode plot.

A further consequence of the control objective is that it might be sufficient to estimate the real plant well up to a certain frequency (somewhere in the region of the cross over) and to tolerate a larger uncertainty for higher frequencies. These aspects move the emphasis from the classical residual analysis to the computation and visualization of the nominal model and the associated uncertainty in the frequency domain.

The main contribution of this paper is to look at three recent concepts delivering a nominal model and the related uncertainty in the Bode plot: the Stochastic Embedding technique [6], Set Membership Identification [4, 11], and Model Error Modelling [8]. The first is a frequency domain

\footnotetext{
${ }^{*}$ This work was supported by the European Union within the European Research Network in System Identification (ERNSI), which is gratefully acknowledged.
} 
method, while the other two work in the time domain. A preliminary objective of our study is to compare the nominal models and their errors - as far as possible in these different frameworks. Our future work will focuss on robust control design, based on these methods.

The paper is organized as follows: In Sections 2-4, we give a quick overview on Stochastic Embedding (SE), Set Membership Identification (SMI) and Model Error Modelling (MEM). We describe basic ideas and give a description of the software, used for carrying out the examples. Finally, in section 5, a comparative example is presented.

\section{Non-Stationary Stochastic Embedding}

\subsection{General Setup}

The idea behind Stochastic Embedding can, somewhat simplified, be described as follows: the True System $G$ can be given as

$$
G(i \omega)=\hat{G}_{0}(i \omega)+\Delta G(i \omega)
$$

where $\hat{G}_{0}$ is a "nominal system" that can be exactly represented within a parameterized family, and $\Delta G(i \omega)$ is a random variable independent of $\hat{G}_{0}$. (This is a turn-around of the conventional interpretation, where the nominal, estimated model

$$
\hat{G}_{0}(i \omega)=G(i \omega)+\Delta G(i \omega)
$$

is seen as the true system plus a model error $\Delta G$, that is independent of $G$.) The true system in (1) is a random variable, which is the root of the term "stochastic embedding". Now, suppose that we have noisy observations of the true system $G$ at certain frequencies:

$$
G_{k}=G\left(i \omega_{k}\right)+\nu_{k}
$$

where $\nu_{k}$ are independent of $G$ and $\Delta G$. Then, combining (1) and (2), gives

$$
G_{k}=\hat{G}_{0}\left(i \omega_{k}\right)+\nu_{k}+\Delta G\left(i \omega_{k}\right)
$$

This is the basic estimation equation, that needs to be complemented with a parameterization of the model $\hat{G}_{0}$ and assumptions about the variance of $\nu_{k}$ and $\Delta G\left(i \omega_{k}\right)$. In [6] it is suggested to use a linear regression parameterization in terms of some orthonormal basis functions $\hat{G}_{0}(\theta)=\mathcal{B} \theta$ and an assumption that $\Delta G$ is such that the relative model error has a variance that increases linearly or quadratically with $\omega$. Then $\Delta G$ can be written as $\mathcal{B} \bar{\theta} \Lambda$ where $\Lambda$ is a random walk process over $\omega$ (this argument being suppressed) and $\bar{\theta}$ comes from prior knowledge. All this leads to

$$
\begin{aligned}
G & =\hat{G}_{0}(\theta)+G_{0}(\bar{\theta}) \Lambda \\
& =\mathcal{B} \theta+\mathcal{B} \bar{\theta} \Lambda .
\end{aligned}
$$

The identification procedure then consists of three main steps:

1. Pointwise least squares estimation of the true transfer function for certain frequencies: therefore, the input $u$ has to be a sum of sinusoids. This step delivers the values $G_{k}$, for certain frequencies. Additionally, statistical properties of the noise $v$ are calculated (i.e. an unbiased estimate of its variance), assuming gaussian noise.

2. Generation of a set of basis functions. 
3. Estimation of parameters in eqn.(5):

(a) Estimation of $\theta$ (called $\hat{\theta}$ ), based on the knowledge of $G_{k}$. Thus, the nominal model $\hat{G}_{0}=\mathcal{B} \hat{\theta}$ in eqn.(4) is the least squares estimate approximation of $G_{k}$ in the subspace spanned by basis functions $\mathcal{B}$.

(b) Statistical properties of the parameter $\hat{\theta}$. As priori knowledge $\bar{\theta}$, the estimate $\hat{\theta}$ is chosen. Thus, eqn.(5) becomes:

$$
G=\mathcal{B} \hat{\theta}(1+\Lambda)
$$

(c) Statistical properties (i.e. an unbiased estimate of its variance) of the random walk, based on the statistical properties of the noise. The random walk $\Lambda$ is a model whose variance of the frequency response increases linearly with frequency up to a certain roll off. The motivation is, that undermodelling (of a pole) can be written as a multiplicative error - cf. eqn.(6).

(d) Quantification of the model error $\mathcal{B} \bar{\theta} \Lambda$ for any frequency, i.e. calculation of its statistical properties.

A variant of the random walk for resonant systems (integrated random walk) is described in [1]: here, the error increases quadratically with the frequency, in order to produce tighter error bounds at low frequencies in the presence of resonant poles.

\subsection{NSSE Package by Julio Braslavsky (May 1999)}

The package provides solutions for all steps mentioned above. In the first step, the least squares estimate is calculated via numerical stable $Q R$ factorization. Numerical problems when using polynomial representations are well known. Therefore, the set of basis functions is represented in the state space, using a zero-pole-gain representation.

For the stochastic embedding of the dynamical error, two different strategies for the random walk are implemented. The uncertainty clouds are displayed in the Nyquist and Bode-plot for a user-defined confidence level.

Regarding the choice of the basis functions, we implicitly have to choose the model order and the basis functions themselves (in the simple case of a Laguerre basis we only have to choose the pole). The correct choice of the model order is a well known problem within identification procedures. Here, additionally, we have to choose the poles, which is not straightforward and produces a nonlinear optimization problem with many local minima. An iterative scheme is suggested using an average modelling error to be minimized:

$$
\sum_{n} \operatorname{trace}\left(\Sigma_{e}\left(\omega_{n}\right)\right) / \# \text { frequencies }
$$

where $\Sigma_{e}\left(\omega_{n}\right)$ denotes the covariance matrix of the model error $\mathcal{B} \bar{\theta} \Lambda$ for each frequency $\omega_{n}$ and the sum in eqn.(7) is taken over either the considered model orders or pole locations. The frequencies $\omega_{n}$ are a discretization of the frequency range of interest (not the frequencies contained in the harmonic input signal). Minimization of this error is performed by fixing one of the two parameters (either order or pole-location), and varying the other one. The roles are changed until the error does no longer decrease significantly. 


\section{Set Membership Identification}

\subsection{General Setup}

Assuming an unknown-but-bounded noise, the set membership approach calculates the feasible set of parameters which are compatible with the assumed model structure and the available data. For example, consider an ARX model

$$
A(q) y(t)=B(q) u(t)+v(t),
$$

linearly parameterized by its polynomial coefficients $\theta:=\left(a_{i}, b_{j}\right)$, and assume $|v(t)| \leq \delta, \forall t$. For given i/o measurements $\left(u_{m}(t), y_{m}(t)\right)$, the feasible set is given by

$$
\Theta=\left\{\theta:-\delta \leq A(q) y_{m}(t)-B(q) u_{m}(t) \leq \delta, \forall t\right\} .
$$

Assuming that the noise bound $\delta$ is given, $\Theta$ turns out to be a polytope in the parameter space. This can be recursively computed, e.g. via the Broman and Shensa algorithm [2], whose computational burden is generally very high. Therefore, to make the problem computationally tractable, approximations by simple geometric forms overbounding the exact feasible set are usually adopted. The classical approach is to construct a decreasing volume sequence of ellipsoids by the Fogel and Huang algorithm [4]. An alternative is the usage of parallelotopes instead of ellipsoids [11,3]. Most of the work centers round the memory reduction in the algorithms and fast convergence.

The result delivered by a SMI routine is the overbounding set and its middle point, the so-called central estimate, which is considered as the nominal model.

Using basis functions. When an OE model structure

$$
y(t)=G(q, \theta) u(t)+v(t), \quad|v(t)| \leq \delta
$$

is considered, the feasible set is not defined by a set of linear inequalities in the model parameters. Thus, in order to reduce the computational burden, the model is parameterized according to a set of basis functions $\mathcal{B}=\left(b_{1}, \ldots, b_{n}\right)$, and the coefficients $\theta=\left(\theta_{1}, \ldots, \theta_{n}\right)^{T}$ of the linear combination $\mathcal{B} \cdot \theta$ are estimated using linear regression. This leads to the setup $y(t)=(\mathcal{B} \cdot \theta) u(t)+v(t),|v(t)| \leq \delta$, whose feasible set is a polytope and can be approximated through the techniques in $[4,11,3]$.

One fundamental difference with respect to the ARX setup is that we restrict ourselves to the subset of the feasible set which is spanned by the basis functions. The SMI routine will deliver a superset of this subset and we will not have the guarantee that the real plant is an element of this set - even if we know the structure of the real plant and the exact noise bound. To overcome this problem, one may increase either the model order or the noise bound (clearly, this bias error occurs for all methods using a parameterization via basis functions). As in the SE setting, choice of model order and basis function pole locations are a crucial step of the identification procedure.

\subsection{SMID Package by Andrea Garulli (June 1999)}

The package provides ellipsoidal and parallelotopic algorithms for SISO ARX models, where the parameters are the coefficients of the polynomials. The Broman and Shensa algorithm for exact calculation of the polytopic feasible set is also implemented. Alternatively, the package provides identification procedures for SISO transfer functions linearly parameterized by basis functions, in order to cover output error structures. The implementation allows Laguerre and Kautz bases, as well as a set of user-defined basis functions. The algorithms come with the central estimate $\theta_{0}$ and the final ellipsoid or parallelotope. Moreover, the frequency uncertainty regions associated to the feasible sets are visualized in the Bode plot. In the OE setup, this is quite natural, whereas the visualization in the ARX case requires the solution of a nonlinear optimization problem. 

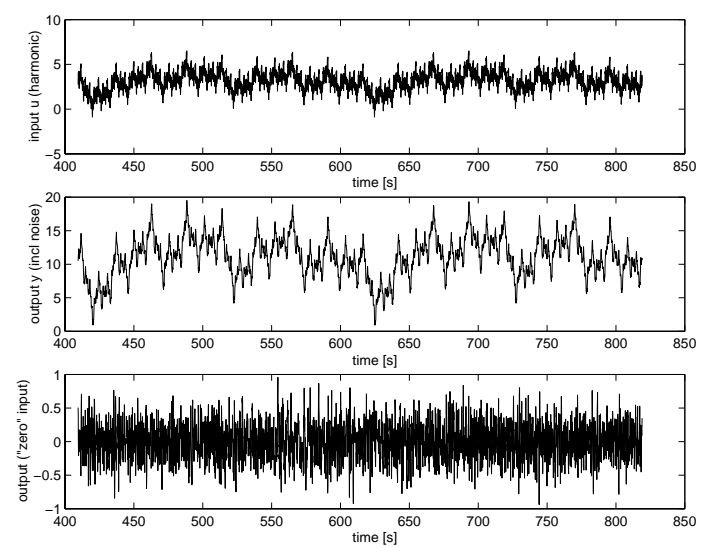

Figure 1: Test input $u$ and output $y$. Below the output of the system using "zero" input.

\section{Model Error Modelling}

We estimate a nominal model $\hat{G}(q)$ of the real plant, for example in an OE setup $y(t)=G(q) u(t)+$ $v(t)$, by an arbitrary estimation method for the given model structure. The classical validation of this model reviews the lags and the crosscorrelation between the residuals and the input. The new idea is to interpret the residual $\epsilon(t)=y(t)-\hat{G}(q) u(t)$ in terms of a model error model

$$
A(q) \epsilon(t)=\frac{B(q)}{F(q)} u(t)+\frac{C(q)}{D(q)} v(t)
$$

and deliver this model error model $(A, B, C, D, F)$ together with its confidence region to the user in the Bode plot [8]. This strategy includes fundamental split between the dynamical error and the noise error, as visible in eqn.(10). The model and its error are validated (better: not invalidated), when zero is included in the model error, i.e. the nominal model belongs to the uncertainty region. As this method is more a new representation of the "classical" identification methods, we can use standard software [9].

\section{Comparative Example}

From the dynamical system with transfer function $G(s)=3.45(s+1) /((0.24 s+1)(0.30 s+1)(6.47 s+$ 1) $\left.\left(0.03 s^{2}+0.32 s+1\right)\right)$ we collect $2 \cdot N(N=4096)$ data points with sampling time $T$ and an input signal consisting of 10 cosinus signals (the excited frequencies are visible in fig. 2 and fig. 3). To avoid initial transients, we only use the time stamps $(N+1) T, \ldots, 2 N T$ for identification. The output is corrupted by noise. To get an idea of the noise, one can think of running the experiment with a zero input. To simulate this situation, we use a white noise input with a low standard deviation (in relation to the test input). All relevant signals are shown in Fig. 1.

\subsection{Identification by Stochastic Embedding}

Preliminary runs for a Laguerre basis (pole $p=-0.1$ ) of order $n=4$ show tight uncertainty regions for low frequencies using the "normal" random walk (see fig. 2). Using the integrated version, we receive of course tighter bounds for low frequencies, but a larger "trumpet" for higher ones. Additionally, the transfer function point estimates (marked by crosses) are not located in the uncertainty region. An unbiased estimate of the variance of the noise, assumed gaussian, is $\hat{\sigma}_{\nu}^{2}=0.0192$ (which is of course independent of the random walk strategy). From this comparison, 

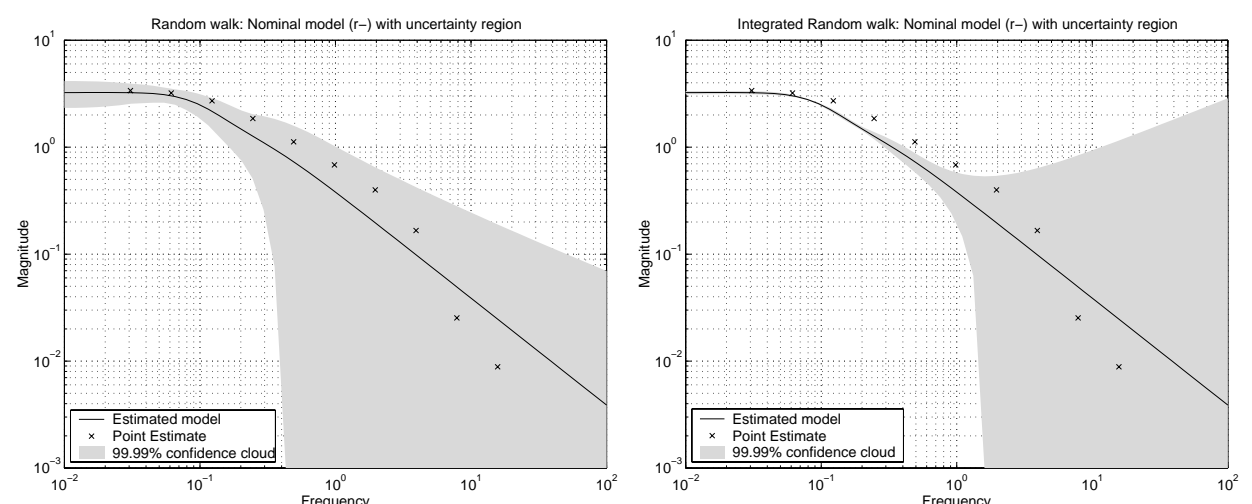

Figure 2: SE: Preliminary comparison between random walk and integrated random walk.

we conclude that the plant does not contain remarkable resonances and proceed with the random walk method.

Starting with this initial order $n=4$, we look for the optimal pole location and obtain $p=-0.32$, using the average modelling error, as suggested in eqn.(7). Proceeding this iterative procedure, we obtain an optimal order $n=6$ for this pole location (now fixed at $p=-0.32$ ). More iterations do not improve the error in eqn.(7), thus the parameters for the Laguerre basis are $n=6, p=-0.32$. The result of the random walk procedure is depicted in fig. 3. The unbiased estimate of the random walk parameter is calculated to $\hat{\sigma}_{\epsilon}^{2}=0.12$. The fit of the nominal model to the transfer function point estimates is somewhat poor and examining the nominal model of order 6 we detect two obvious pole-zero cancellations, which leads us, despite the suggested optimality, to the final decision to choose a fourth order model.

Looking at the transfer function point estimates, we expect system poles at about $-0.1,-2$, -20 , and therefore adjust the fourth order Laguerre basis with optimal pole at $p=-0.32$ by replacing elements $b_{2}(s), b_{3}(s), b_{4}(s)$ by

$$
b_{2}(s)=\frac{1}{s+0.1}, b_{3}(s)=\frac{1}{s+2}, b_{4}(s)=\frac{1}{s+20} .
$$

Running the integrated random walk we obtain the modelling parameters

$$
\hat{\theta}=(0.68,-1.42,-0.27,0.21)
$$

together with an unbiased estimate of the random walk parameter of $\hat{\sigma}_{\epsilon}^{2}=0.03$. The nominal transfer function is given finally by

$$
\hat{G}_{S E}(s)=\frac{-0.9349 s^{3}+7.851 s^{2}+29.38 s+4.607}{s^{4}+22.42 s^{3}+49.38 s^{2}+17.7 s+1.299}
$$

and depicted together with its uncertainty region in fig. 3. All in all, we received a fourth order nominal model together with tight error bounds up to frequencies $\omega \approx 2$. The choice of the parameters is basically straightforward due to the suggested average modelling error. The choice of arbitrary basis functions allows the incorporation of additional knowledge received from the point estimates at the excited frequencies. Finally, we remark that we used the $99 \%$ confidence level for a better comparison to the other two methods. The qualitative results using a $90 \%$ level are the same, receiving of course tighter bounds, especially for low frequencies. 

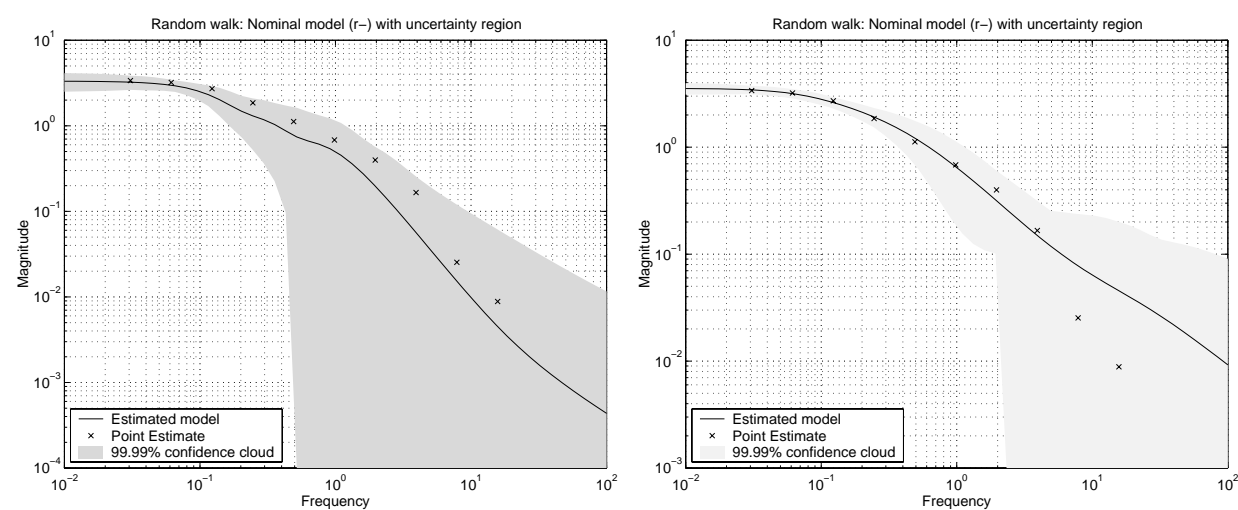

Figure 3: SE: Nominal model and uncertainty region using 6th order Laguerre basis (upper plot) and adjusted 4 th order basis (lower plot).

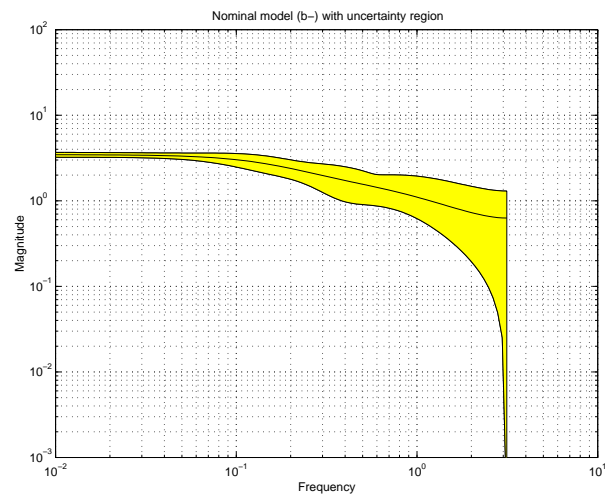

Figure 4: SMI: 4th order Kautz base with poles 0.84, 0.53 ( $\delta=1.5$, final volume 0.0037$)$.

\subsection{Identification by Set Membership Identification}

Having an Output Error model, we use the representation of the nominal model by basis functions. Because of a higher flexibility, we use a Kautz basis instead of a Laguerre basis. The overbound of the feasible set is calculated using the ellipsoidal algorithm.

Starting with a Kautz basis with parameters ${ }^{1}(0.95,-0.85)$ (i.e. poles at $\left.0.88 \pm 0.28 i\right)$, order $n=4$ and a noise bound of $\delta=1$ (as suggested by the "zero input" experiment), we receive an empty intersection. Suppose, we overestimated the real noise bound, this empty intersection is now due to an underestimation of the real plant order or a fundamental error in the choice of the Kautz parameters. After some preliminary studies, Kautz parameters have been changed to $(0.95,-0.45)$, which means a shift of the Kautz poles to $0.85,0.53$, i.e. from complex to real ones. In order to obtain a nonempty feasible set, it turns out that the noise bound has to be increased to about 1.5. The obtained nominal model together with the Bode plot uncertainty region, are depicted in fig. 4 . The nominal model is

$$
G_{S M I}(z)=\frac{0.98(z-0.84)\left(z^{2}-1.34 z+0.46\right)}{(z-0.85)^{2}(z-0.53)^{2}}
$$

i.e. the Kautz functions are linearly combined with parameters $\hat{\theta}=(1.06,0.69,0.076,0.028)$.

\footnotetext{
${ }^{1}$ Kautz pair $(b, c)$ means a denominator of $\left(z^{2}+b(c-1) z-c\right)^{m / 2}$ for the Kautz function of order $m$.
} 

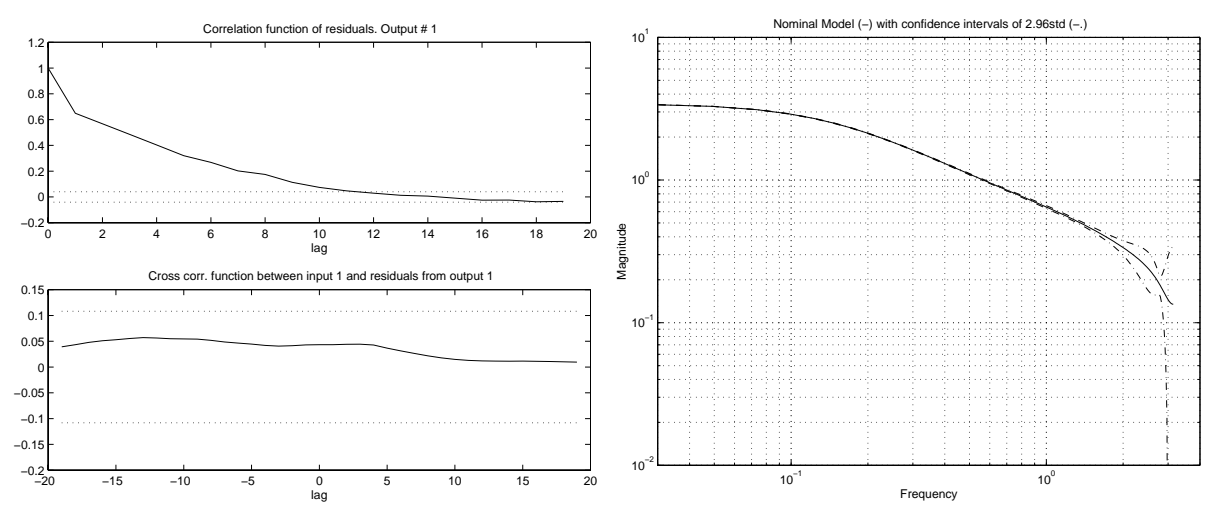

Figure 5: MEM: correlation function (upper plot), cross correlation (middle plot), and confidence region (lower plot) for the $O E(4,4,0)$ nominal model.
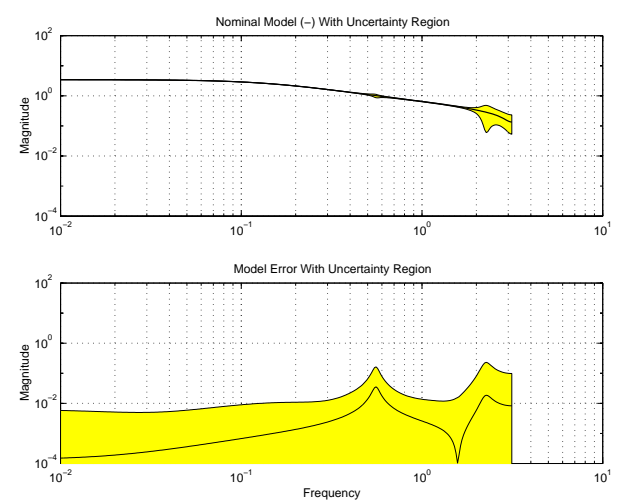

Figure 6: MEM: $O E(4,4,0)$ nominal model together with uncertainty region as provided by a $(5,6,8,0,0)$ error model (upper plot) and the error model error (lower plot).

\subsection{Identification by Model Error Modelling}

The production of the nominal model in the first step is independent of the error model, thus we calculate the prediction error estimate of an output-error model $n_{f}=4, n_{b}=4, n_{k}=0$ (or short: $O E(4,4,0)$, see [9]). The residual analysis and the "classical" confidence region are depicted in fig. 5. The nominal model delivered is

$$
G_{M E M}(z)=\frac{0.48 z^{3}+0.21 z^{2}-0.13 z-0.01}{z^{4}-0.65 z^{3}-0.29 z^{2}+0.12 z-0.02}
$$

We start with the design of the model error model with a low order model. Finally, for the parameter set $n_{a}=5, n_{b}=6, n_{c}=8, n_{d}=0, n_{f}=0$, we receive a not falsified error model with tight bounds, nearly over all frequencies, as shown in fig. 6 .

\subsection{Comparison}

As result of the three identification procedures, we receive the nominal models given by eqns. $(11,12,13)$ and the uncertainty regions shown in figs. 3,4,6.

Note, that none of the uncertainty regions is a "100\%-region". SE and MEM produce statistical regions "by construction". In the case of SMI, this appears because of possible undermodelling of the plant or underestimation of the noise bound and the parameterization via basis functions. All three methods end up with a fourth order model, the amplitudes are shown in fig. 7. Notable, that 


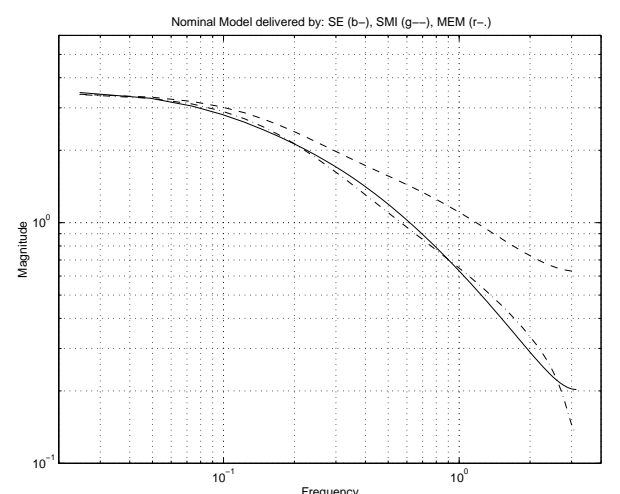

Figure 7: Bode plots of all nominal models.
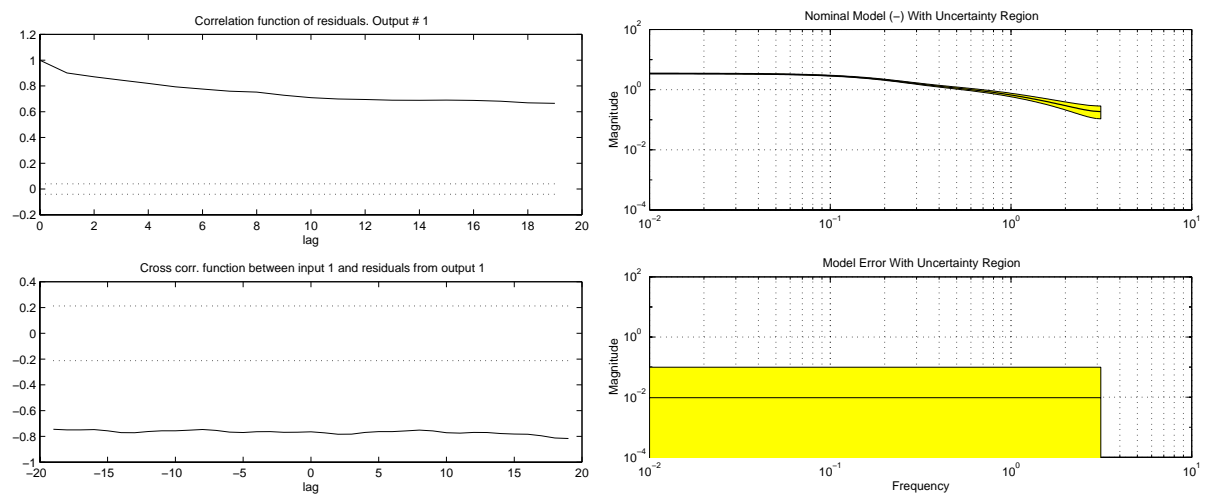

Figure 8: SE: residuals and $(0,1,8,0,0)$ model error model.

SMI produces a "nearly $3 r d$ order" model due to the pole-zero cancellation, visible in eqn.(12). This explains the difference between the magnitude at higher frequencies of the SE and MEM nominal model on the one hand and SMI model on the other hand.

Looking at the shape of the uncertainty regions, the uncertainty grows with frequency, but seem to be acceptable for a robust controller design. We did not display the real plant in this example session, but we point out, that it is contained in the uncertainty regions of all final designs.

In order to compare all methods according to one criterion, we run the MEM technique for the nominal models, that are delivered by SE and SMI. Figs. 8 and 9 show the results. The SE nominal model can be "non-falsified" with a low order model error model, ending up in nearly the same small uncertainty region as MEM itself. But as SE and MEM nearly provide the same nominal model, it is not surprising. Running the SMI nominal model in the MEM framework, it ends up with a high order model error model and a larger uncertainty region, compared with the other methods and the uncertainty region delivered by SMI. This is not surprising due to the difference in the nominal models.

\section{Conclusions and Future Works}

We compared identification methods, working in the time and frequency domain and following statistical or deterministic philosophy. Due to these different nature a fair comparison is quite difficult. Moreover, application of one of the methods will heavily depend on the a priori knowledge (for example, if harmonic input signals are possible or a priori information on the noise amplitude or statistics are available). However, we showed the main features of the methods and obtained 

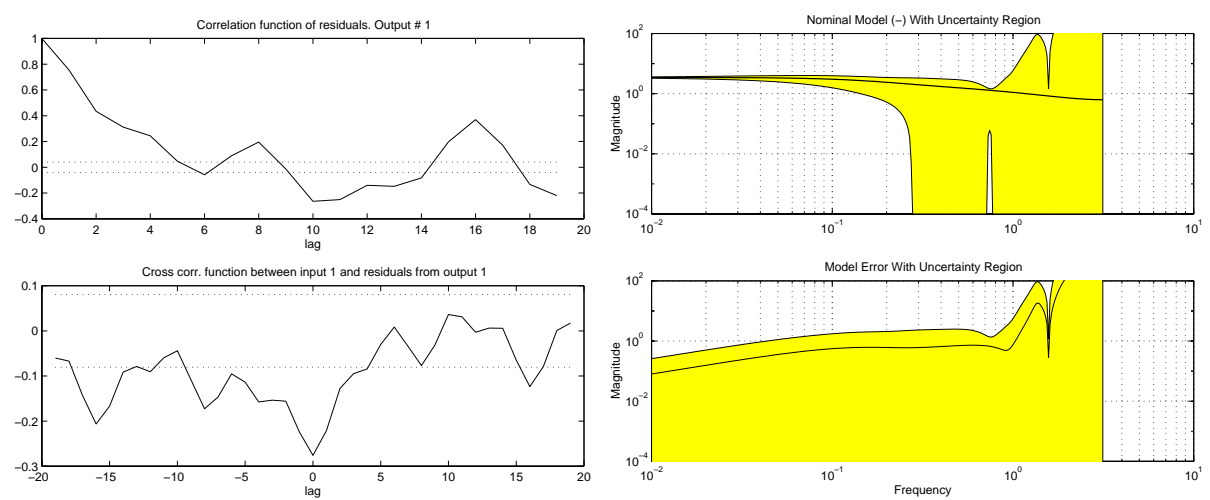

Figure 9: SMI: residuals and $(8,10,25,10,10)$ model error model.

reliable nominal models and acceptable related uncertainties in all three cases. As the methods are based on different philosophies, fruitful combinations are possible. For example, one might think of providing a rough estimation of system poles from a frequency pointwise estimation (as necessary for Stochastic Embedding) to the other methods. Also, the fact that dynamical error and noise error is not explicitly separated within Set Membership techniques, will be an aim for future activities.

\section{References}

[1] J. H. Braslavsky and G. C. Goodwin. A note on non-stationary stochastic embedding for modelling error quantification in the estimation of resonant systems. Technical Report EE99014, CIDAC, Dept of Electrical Engineering, Univ of Newcastle, Callaghan, Australia, Jan. 1999.

[2] V. Broman and M. J. Shensa. A compact algorithm for the intersection and approximation of n-dimensional polytopes. Mathematics and Computers in Simulation, 32:469-480, 1990.

[3] L. Chisci, A. Garulli, A. Vicino, and G. Zappa. Block recursive parallelotopic bounding in set membership identification. Automatica, 34:15-22, 1998.

[4] E. Fogel and F. Huang. On the value of information in system identification - bounded noise case. Automatica, 18(12):229-238, Dec. 1982.

[5] A. Garulli, A. Tesi, and A. Vicino, editors. Robustness in Identification and Control. Number 245 in Lecture Notes in Control and Information Sciences. Springer-Verlag, 1999.

[6] G. C. Goodwin. Identification and robust control: Bridging the gap. In Proc. of the 7th IEEE Mediterranian Conference on Control and Automation, Haifa, Israel, June 1999.

[7] R. L. Kosut, G. C. Goodwin, and M. P. Polis. Special issue on system identification for robust control design. IEEE Trans. on Automatic Control, 37(7), July 1992.

[8] L. Ljung. Model validation and model error modeling. In B. Wittenmark and A. Rantzer, editors, Proc. of the Aström Symposium on Control, pages 15-42, Lund, Sweden, Aug. 1999. Studentliteratur, Lund, Sweden.

[9] L. Ljung. System Identification Toolbox - for use with MATLAB. The Mathworks Inc., Natick, MA, USA, 2000. 
[10] T. Söderström and K. J. Åström. Special issue on trends in system identification. Automatica, 31(17), Dec. 1995.

[11] A. Vicino and G. Zappa. Sequential approximation of feasible parameter sets for identification with set membership uncertainty. IEEE Trans. on Automatic Control, 41(6):774-785, June 1996. 